\title{
CagA, A Master Regulator of Host Protein Phosphorylation Events
}

\author{
Markus Stein* \\ Department of Health Sciences, Albany College of Pharmacy and Health Sciences, Albany, USA
}

\begin{abstract}
Received: December 02, 2013; Accepted: December 21, 2013; Published: J anuary 02, 2014
*Corresponding author: Markus Stein, Department of Health Sciences, Albany College of Pharmacy and Health Sciences 106, New Scotland Avenue, USA, Tel: 518-694-7174; Fax: 518-694-7382; E-mail: markus.stein@acphs.edu
\end{abstract}

Among the many pathogenic bacteria, Helicobacter pylori $(H$. pylori) is the only one that is widely recognized as a causative agent of human cancer, specifically adenocarcinoma of the stomach [1-3]. Bacterial infection is thought to increase the risk of cancer development by a factor of approximately six-fold, although genetic predispositions of the host, including promoter mutations in pre-inflammatory genes, play an important role in cancer development in addition to infection. Many virulence factors of $H$. pylori are important for colonization of the human stomach and for manipulating the immune response and allow the bacterium to cause chronic infection. However, the major cancer-associated bacterial factor is likely the CagA cytotoxin, which has been shown to act as an oncogene in animal models [4]. Following bacterial attachment to the gastric epithelium, a complex type IV secretion system is translocating CagA into the host epithelial cell [5-8]. A myriad of studies have investigated the role of CagA on a molecular level and provided clues as to how it might induce gastric malignancy. From these studies it has become clear that CagA acts as a multifunctional molecule that interacts with many host proteins that are involved in host cell signaling. While many excellent reviews discussing the various CagA functions are available $[9,10,2,11]$ I would like to discuss in this editorial one specific function of CagA, which appears to be a major theme of CagA activities and this is its ability to alter the function of host kinases and phosphatases. Phosphorylation events regulate a myriad of cellular signaling pathways and are therefore ideal targets of bacterial virulence factors such as CagA that aim to modify host response without killing the host.

Early on in CagA research it was observed that the presence of CagA caused a strongly reduced tyrosine- phosphorylation of a set of host proteins [12]. Later studies showed that this reduction was caused by two CagA-dependent mechanisms. The first mechanism involved the catalytic inhibition of c-src-kinases causing the dephosphorylation of proteins including cortactin [13], ezrin [14] and vinculin [15], which cause the rearrangement of the actin cytoskeleton and disruption of matrix adhesion. Interestingly, tyrosine-phosphorylation of CagA (CagA $\left.{ }^{\mathrm{p}-\mathrm{Tyr}}\right)$ on a c-terminal motif (EPIYA) by Src-family kinases or c-Abl kinase was required for CagA activation $[16,17]$ and its ability to inhibit Src-kinase thereby engaging in a negative feedback loop regulating CagA phosphorylation status and activity [18]. The second mechanism of host protein dephosphorylation was defined by the ability of activated CagA ${ }^{\mathrm{p}-\mathrm{Tyr}}$ to bind to the SH2domain of Shp-2 tyrosine phosphatase [19], which triggered the dephosphorylation of focal adhesion kinase (FAK)and as a consequence of FAK substrates like Paxillin [20]. In gastric epithelial cells reduced FAK activity leads to a reduction of focal adhesion sites and contributes to cell detachment and increased cell motility.

While dephosphorylation of proteins on tyrosine residues appears important for promoting detachment of epithelial cells from the extracellular matrix, CagA-mediated inhibition of the cellular serine-threonine kinase Par1b/MARK2 was shown to be crucial for disruption of apical-basolateral cell polarity $[21,22]$. Par1b/MARK2 inhibition was independent of CagA activation via tyrosine phosphorylation and required the carboxyterminal CagA multmerization motif (CM - FPLKRHDKVDDLSK). This CagA motif was shown to act as a structural analogue of Par1b/ MARK2 substrates and it binds to the substrate binding pocket of the kinase, thereby inhibiting its activity [23]. Par1b/ MARK2 inhibition leads to reorganization of microtubules and breakdown of tight junctions. Furthermore, Par1b/MARK2 inhibition also affected cortical actin and stress fiber formation and cell motility by preventing the inactivation of the RhoA specific guanosine exchange factor GEF-H1, thus synergizing with CagA ${ }^{\mathrm{p}-\mathrm{Tyr}}$ dependent functions [24].

The CM motif is also involved in direct binding to E-cadherin thereby preventing the association of E-cadherin with $\beta$-catenin. This destabilizes the E-cadherin- $\beta$-catenin complex and causes redistribution of $\beta$-catenin to the nucleus where it initiates the transcription of cancer-related genes [25]. Since serinephosphorylation of $\beta$-catenin targets the molecule for degradation via the proteasome and prevents nuclear localization, CagA seems to synergize with other host signaling pathways to prevent $\beta$-catenin phosphorylation and degradation. Additionally, CagA was shown to induce the $\beta$-catenin pathway by interacting with c-Met and activation of the c-Met associated PI3K-AKT signaling pathway [8].

In conclusion, CagA perturbs host phosphatase- and kinase- 
signaling pathways that regulate processes that have been shown to be important in epithelial-to-mesenchymal transition and tumor formation by disrupting cellular adhesion to the extracellular matrix, cadherin-mediated cell adhesion and tight junction formation. Together these processes may lead to an increased risk for developing gastric cancer and strengthen the hypothesis of CagA as the only bacterial oncogene to date.

However, many questions concerning CagA function in $H$. pylori associated gastric diseases remain unanswered. One daunting question is why some strains of Helicobacter have acquired the type IV secretion system during evolution and translocate the CagA effector. Cancer development and host mortality is clearly an undesired outcome from the bacterial perspective and a result not only of CagA activity; but also of other mechanisms, most notably chronic bacterial inflammation. For this reason, a possible physiologic advantage of CagAtranslocating strains remains somewhat elusive, despite intense research and much progress in the field. Interestingly, $H$. pylori strains lacking the type IV secretion system and CagA are perfectly able to colonize the human stomach and cause chronic infection, albeit inflammatory responses are significantly reduced during infection with such strains. Therefore, suggesting a role for CagA as a colonization factor is also not undisputed. Nevertheless, the hypothesis that $H$. pylori uses CagA to gain more efficient access to nutrients from host tissue exudates, rather than from food content in the gastric lumen remains attractive. In agreement with this hypothesis, cancer is likely an accidental event, which is the long-term result of CagA activity, chronic gastric inflammation, and additional contributing factors. It should be stated that only a minority of those infected develop gastric disease and even fewer develop gastric cancer, indicating a role for host genetic variations. Clearly, the presence of strains expressing the type IV secretion system and efficiently translocating CagA into the host is an important factor, but not sufficient per se to trigger severe disease in every infected individual. Without doubt many interesting questions concerning $H$. pylori virulence and CagA function remain to be answered.

\section{Acknowledgement}

Markus Stein is supported by a National Institute of Health Grant (\#1R01CA160790-01A1).

\section{References}

1. Peek Jr RM, Blaser MJ (2002) Helicobacter pylori and gastrointestinal tract adeno carcinomas. Nat Rev Cancer, 2(1): 28-37.

2. Polk DB, Peek Jr RM (2010) Helicobacter pylori: gastric cancer and beyond. Nat Rev Cancer, 10(6): 403-414.

3. Wroblewski LE, Peek Jr RM (2013) Helicobacter pylori in gastric carcinogenesis: mechanisms. Gastroenterol Clin North Am, 42(2): 285298.

4. Ohnishi N, Yuasa H, Tanaka S, Sawa H, Miura M, et al. (2008) Transgenic expression of Helicobacter pylori CagA induces gastrointestinal and hematopoietic neoplasms in mouse. Proc Natl Acad Sci USA, 105(3): 1003-1008.
5. Segal ED, Cha J, Lo J, Falkow S, Tompkins LS (1999) Altered states: involvement of phosphorylated CagA in the induction of host cellular growth changes by Helicobacter pylori. Proc Natl Acad Sci, USA 96(25): 14559-14564.

6. Stein M, Rappuoli R, Covacci A (2000) Tyrosine phosphorylation of the Helicobacter pylori CagA antigen after cag-driven host cell translocation. Proc Natl Acad Sci USA, 97(3): 1263-1268.

7. Odenbreit S, Püls J, Sedlmaier B, Gerland E, Fischer W, et al. (2000) Translocation of Helicobacter pylori CagA into gastric epithelial cells by type IV secretion. Science, 287(5457): 1497-1500.

8. Suzuki M , Mimuro H, Kiga K, Fukumatsu M, Ishijima N, et al. (2009) Helicobacter pylori CagA phosphorylation-independent function in epithelial proliferation and inflammation. Cell Host Microbe, 5(1): 2334.

9. Hatakeyama M (2004) Oncogenic mechanisms of the Helicobacter pylori CagA protein. Nat Rev Cancer, 4(9): 688-694.

10. Hatakeyama M (2008) SagA of CagA in Helicobacter pylori pathogenesis. Curr Opin Microbiol, 11(1): 30-37.

11. Tegtmeyer N, Wessler S, Backert S (2011) Role of the cag-pathogenicity island encoded type IV secretion system in Helicobacter pylori pathogenesis. FEBS J, 278(8): 1190-1202.

12. Püls J, Fischer W,Haas R (2002) Activation of Helicobacter pylori CagA by tyrosine phosphorylation is essential for dephosphorylation of host cell proteins in gastric epithelial cells. Mol Microbiol, 43(4): 961-969.

13. Selbach M, Moese S, Hurwitz R, Hauck CR, Meyer TF, Backert S (2003) The Helicobacter pylori CagA protein induces cortactin dephosphorylation and actin rearrangement by c-Src inactivation. EMBO J, 22(3): 515-528.

14. Selbach M, Moese S, Backert S, Jungblut PR, Meyer TF (2004) The Helicobacter pylori CagA protein induces tyrosine dephosphorylation of ezrin. Proteomics, 4(10): 2961-2968.

15. Moese S, Selbach M, Brinkmann V, Karlas A, Haimovich B, Backert S, Meyer TF (2007) The Helicobacter pylori CagA protein disrupts matrix adhesion of gastric epithelial cells by dephosphorylation of vinculin. Cell Microbiol, 9(6): 1148-1161.

16. Stein M, Bagnoli F, Halenbeck R, Rappuoli R, Fantl WJ, Covacci A (2002) c-Src/Lyn kinases activate Helicobacter pylori CagA through tyrosine phosphorylation of the EPIYA motifs. Mol Microbiol, 43(4): 971-980.

17. Poppe M, Feller SM, Römer G, Wessler S (2007) Phosphorylation of Helicobacter pylori CagA by c-Abl leads to cell motility. Oncogene, 26(24): 3462-3472.

18. Tsutsumi R, Higashi H, Higuchi M, Okada M, Hatakeyama M (2003) Attenuation of Helicobacter pylori CagA x SHP-2 signaling by interaction between CagA and C-terminal Src kinase. J Biol Chem, 278(6): 3664-3670.

19. Higashi H, Tsutsumi R, Muto S, Sugiyama T, Azuma T, et al. (2002) SHP-2 tyrosine phosphatase as an intracellular target of Helicobacter pylori CagA protein. Science, 295(5555): 683-686.

20. Tsutsumi R, Takahashi A, Azuma T, Higashi H, Hatakeyama M (2006) Focal adhesion kinase is a substrate and downstream effector of SHP-2 complexed with Helicobacter pylori CagA. Mol Cell Biol, 26(1): 261276.

21. Saadat I, Higashi H, Obuse C, Umeda M, Murata-Kamiya N, et al. (2007) Helicobacter pylori CagA targets PAR1/MARK kinase to disrupt epithelial cell polarity. Nature, 447(7142): 330-333. 
22.Zeaiter Z, Cohen D, Müsch A, Bagnoli F, Covacci A, et al. (2008) Analysis of detergent-resistant membranes of Helicobacter pylori infected gastric adenocarcinoma cells reveals a role for MARK2/Par1b in CagAmediated disruption of cellular polarity. Cell Microbiol, 10(3): 781-794.

23. Nesić D, Miller MC, Quinkert ZT, Stein M, Chait BT, et al. (2010) Helicobacter pylori CagA inhibits PAR1-MARK family kinases by mimicking host substrates. Nat Struct Mol Biol, 17(1): 130-132.

24. Yamahashi Y, Saito Y, Murata-Kamiya N, Hatakeyama M (2011) Polarity- regulating kinase partitioning-defective $1 \mathrm{~b}$ (PAR1b) phosphorylates guanine nucleotide exchange factor H1 (GEF-H1) to regulate RhoAdependent actin cytoskeletal reorganization. J Biol Chem, 286(52): 44576-44584.

25. Murata-Kamiya N, Kurashima Y, Teishikata Y, Yamahashi Y, Saito Y, et al. (2007) Helicobacter pylori CagA interacts with E-cadherin and deregulates the beta-catenin signal that promotes intestinal trans differentiation in gastric epithelial cells. Oncogene, 26(32): 46174626. 